\title{
Buying Time for Better Decision-Making: The Impact of Home Based Re- habilitation on Frail Older People
}

\author{
D. Scott* and M. Donnelly \\ Centre for Clinical and Population Sciences, Queen's University, Royal Victoria Hospital, Belfast BT12 6BJ
}

\begin{abstract}
Often, life-changing decisions regarding the long-term care needs of frail older patients are taken at vulnerable times when they are ill or hospitalized. Consequently, patients may be placed inappropriately in institutional care where it is difficult to regain the skills or confidence needed to return home. This paper presents findings from an evaluation of a service that extends the period of decision making about patients needs by providing a rehabilitative package of care in the patient's own home. Participants comprised two groups; older people who received the rehabilitation service $(n=56)$ and a comparator group of similar older people who received a care managed service $(n=56)$. All participants completed a series of measures at three time-points. These measures included an assessment of functioning, a measure of quality of life and, a measure of service utilization. On discharge, rehabilitation clients experienced significant improvements relative to caremanaged clients on all functional measures (FIM, $\mathrm{P}=.014$; CDI, $\mathrm{P}=.001$; EASY, $\mathrm{P}=.001$ ); quality of life ( $\mathrm{P}=.003)$; and, service utilization and cost $(\mathrm{P}=.001)$. In addition, carers of rehabilitation service users reported significantly less stress than carers of care-managed patients $(\mathrm{P}=.007)$. These results show that the rehabilitation service succeeded in improving functioning and helped to create a longer time-period during which older people, their carers and professionals could make appropriate decisions about their future care needs.
\end{abstract}

\section{INTRODUCTION}

Major decisions about the long-term care needs of older people are often taken at inappropriate times in their life when they are ill or in an unfamiliar setting such as hospital (Wright, 2003). Indeed, some researchers suggest that professional decision-making regarding the future care of frail older people does not consider that an older person may have the potential to improve (Clisset, 2001). As a consequence, patients may be placed inappropriately in institutional care where it can be difficult to regain the skills and confidence necessary to return home (Reed \& Morgan, 1990; Giustis et al., 2006; Vincent et al., 2007).

One of the aims of the policy of 'intermediate care' (Thomas \& MacMahon, 2001; Melis et al., 2004) is to avoid the unnecessary or premature placement of older people in long-term residential or nursing care. This policy seeks to ensure that frail or ill older people have an opportunity to receive a period of rehabilitation that will help them realize their full potential and improve the appropriateness of decisions regarding their future care needs (NHS, 2000; NHS, 2003). A range of intermediate care services has developed across the United Kingdom.

This paper presents the findings of an evaluation of a home-based rehabilitation service for elderly people who were at the 'threshold' of entry to care management. Overall, the service was designed to develop better decision-making regarding the appropriate long-term service requirements and placement of frail older people.

*Address correspondence to this author at the Centre for Clinical and Population Sciences, Queen's University, Royal Victoria Hospital, Belfast BT12 6BJ; E-mail: david.scott@qub.ac.uk

\section{Description of the Rehabilitation Service}

The primary aim of the service is to develop a community-based rehabilitation service for frail older people who are on the verge of receiving a care-managed service. The rehabilitation service provides a holistic and comprehensive assessment of functioning and, where appropriate, a period of care and rehabilitation in the client's own home before their longer-term care needs are determined. The rehabilitation service has grown since its inception and currently consists of one part-time manager ( 0.5 WTE), physiotherapy (1.75 WTE), with additional input from a 'junior' physiotherapist, occupational therapy (1 WTE) and ten rehabilitation assistants. These assistants come from a variety of backgrounds; some have previously worked in the Trust's Intensive Home Care Service while others have worked for caring organizations in the private sector. All rehabilitation assistants receive training in such areas as manual handling and skin care. They also receive 'on the job' training from the physiotherapist and occupational therapist in the care and rehabilitation of each individual service user.

The majority of referrals to the service are hospital inpatients and the main referral agent is the Trust's Care Management Team. Upon receiving a referral the physiotherapist and occupational therapist (key therapists) complete a comprehensive assessment using validated assessment tools. The assessment considers the type of care and therapy previously provided to the client as well as discussing the clients' own rehabilitation goals and aspirations. In addition, the occupational therapist completes an assessment of the service user's home environment particularly their kitchen and bathroom; they will then supply any equipment or adaptations necessary to allow the client to be safely rehabilitated in their own home. The physiotherapist produces a written care and reha- 
bilitation plan based on the individual goals and needs of each client. This plan is completed in conjunction with the client who is provided with a written copy. The plan includes information about the number of home visits the client will receive and details the types of activities that will be undertaken by the rehabilitation team during each visit.

During initial visits the 'key therapist' is present to supervise the completion of the rehabilitation activities and to ensure that clients and rehabilitation assistants use any equipment safely and effectively. This supervisory role is gradually reduced as assistants and service users become more competent in the completion of activities of daily living, rehabilitation related tasks and the use of equipment. The types of activities undertaken by the rehabilitation service vary between service users but commonly include; a specific exercise plan to increase mobility and strength as well as assisting service users to complete activities of daily living (ADLs) as independently and safely as possible. These ADLs typically include getting in and out of bed, transferring to a toilet or commode, washing/showering and meal preparation. Activities of daily living are seen as opportunities to facilitate the rehabilitation process and it is hoped that the confidence of service users will increase through the successful completion of these tasks.

The rehabilitation team meets weekly to discuss the progress of each client. The key therapists meet with each client regularly to assess and discuss their progress. As service users become more competent in the completion of tasks their care plan is amended and the nature and level of assistance provided is reduced accordingly. On average, the rehabilitation service is provided for a maximum of thirteen weeks. However, in a small number of cases (7) clients remained on the scheme for a longer period as they were continuing to make good progress. Clients are discharged from the service when they are able to function independently or when their functioning has reached a plateau and no further progress is likely, for the majority of clients this occurs within a thirteen week period. Prior to discharge the key therapists will again complete a functional assessment and provide a full report to the client's care manager detailing their current level of functional ability. If necessary, the care manager will then provide services appropriate to the long term care needs of each patient.

This paper examines and evaluates the extent to which the rehabilitation service has met its stated objectives in terms of increasing or restoring functioning and independence; improving the quality of life of service users and their carers; and, reducing or 'shifting' care management expenditure.

\section{METHODOLOGY}

Ethical approval for the study was provided by the Trust's Research Ethics Committee. The study employed a prospective repeated measures design with a comparator group. The comparator group was made up of older people who were receiving a standard or 'usual' care managed service. These frail older people had an impaired capacity for self-care as a result of hospitalization, disease or trauma and were unable to independently complete a range of daily living functions. The care management service provided care to assist these older people in activities of daily living and maintain them in their current living situations. It should be noted that all comparator group participants would have been eligible for the rehabilitation service had there been a place available at the time of their entry to care management. Study participants were interviewed at three time-points; upon entry to the service, eight to ten weeks later and, six months following their initial placement. Rehabilitation service clients were recruited by a researcher upon entry to the service. All new service users were approached and those who provided their written informed consent were admitted to the study. Comparator group participants were recruited directly from the Trusts' Care Management Team. A researcher approached care managers on a weekly basis and information was collected on new referrals who were eligible for the rehabilitation service but for whom no place was available. A researcher contacted these clients and the nature of the study was explained to them before they were asked to provide their written informed consent to participate in the study. Those care management clients who agreed to participate received an evaluation from a physiotherapist to ensure that they would have been eligible for the rehabilitation service had a place been available. Those clients who would not have been eligible for the rehabilitation service were not admitted to the study.

Participants completed a series of measures at each timepoint. Functional status was measured using the Functional Independence Measure (FIM) (Keith et al., 1987), Community Dependency Index (CDI) (Eakin \& Baird, 1995) and EASY-Care questionnaire (Northern Ireland version) (Philp et al., 1998). All these measures are designed to be completed by a trained health professional and were completed by a physiotherapist. A researcher interviewed each participant using the Short-Form-36 (SF-36) (Ware et al., 1992) measure of health status and the Patient Generated Index (PGI) (Dempster \& Donnelly, 2000) of quality of life. Service utilization was measured using the Client Services Receipt Inventory (CSRI) (Beecham \& Knapp, 1992) while associated costs were calculated using PSSRU unit costs (Netten \& Curtis, 2003).

In addition, a total of 49 clients who agreed to participate had a carer and 46 provided their written informed consent and agreed to complete the Carers Strain Index (CSI) (Robinson, 1983) and the Carers' Generated Index of Quality of Life (CGI) (Audit Commission, 1997). However, fewer carers were available to complete these measures at the second time-point $(n=38)$ and the final interview $(n=31)$.

\section{RESULTS}

\section{Rehabilitation Service Activity 1999-2004}

Overall, between July 1999 and December 2004 the rehabilitation service provided care to 187 older people. On average, service users were 83 years old and the majority were female $(149 / 187,79 \%)$. More than three-quarters of clients were referred to the rehabilitation service from hospital $(146 / 187,78 \%)$. On average, rehabilitation service users had spent 96 days in hospital however there was considerable variation in the length of inpatient stay (between 6 and 287 days). 
An analysis of patient records showed that on average, clients received the rehabilitation service for 6.7 weeks (range: one week to sixteen weeks) During this time rehabilitation assistants provided an average of 102.91 hours of care for each client (range 21 hours to 280 hours). Each client also received on average 7.3 visits from a physiotherapist (range 1 to 27 visits) and six visits from an occupational therapist (range 2 to 27 visits). The majority of older people who used the rehabilitation service during this period had a restricted level of functional ability as measured by the FIM, CDI and EASY-Care scales. This restriction in functional ability was the result of a wide range of conditions.

\section{PROFILE OF A SAMPLE OF SERVICE USERS AND USERS OF 'USUAL' SERVICES}

The general characteristics of patients receiving either the rehabilitation service $(n=56)$ or users of 'usual care $(n=56)$ at the time of admission to these services were similar. There were no statistically significant differences between the two groups in terms of sex or age (median average age of the rehabilitation group was 73.5 years vs. 73 years in the usual care group).

\section{Functional Ability}

There were no differences between rehabilitation users and users of usual services with respect to functional ability at the time of admission. Rehabilitation service users' experienced statistically significant improvements relative to usual service users on all functional measures at the first follow-up point (see Table 1). This significant difference was again noted for the EASY measure during the six month follow-up interview $(\mathrm{t}(1.92)=-2.87, \mathrm{P}=.005)$. Functional scores recorded using the FIM and CDI found no significant differences between the two groups at the final time-point.

The change within each group during the course of the study was also examined. Rehabilitation service users experienced statistically significant improvements in functioning on all three scales during the six-month period (FIM, Wilks' Lambda $=.354(2,48)=43.83, \mathrm{P}=.001)$; CDI, Chi Sq. $(2,48)$ $=34.599, \mathrm{P}=.001$; EASY, Wilks Lambda $=.371(2,48)=$ $40.67, \mathrm{P}=.001)$. The functional scores of usual service users also improved during the course of the study though this change was statistically significant for functioning as recorded using the CDI only (Chi Sq. $(2,48)=10.08, \mathrm{P}=.006)$. In order to examine the possibility that variances in functional impairment scores at the time of admission were having an impact on functional scores recorded at the other time points an Analysis of Co-Variance (ANCOVA) was conducted with baseline scores entered as the co-variate. The dependent scores were FIM scores at the eight - ten week and six-month time point (CDI scores were not normally distributed and therefore did not meet the assumptions necessary to perform an ANCOVA). Having controlled for variance between the two groups at baseline the ANCOVA demonstrated that there was still a significant difference between the two groups when they were assessed eight - ten weeks later and again at six months $(\mathrm{F}(1,91)=23.9, \mathrm{P}=.001)$. The EASY measure also met the assumptions for ANCOVA and yielded a similar result $(\mathrm{F}(1,91)=36.01, \mathrm{P}=.001)$.

\section{Health Status}

Health status was assessed using the SF-36. There were no significant between group differences at any time point during the study when rehabilitation users and usual service users were compared on any of the measures eight subscales (physical functioning, role physical, bodily pain, general health, vitality, social functioning, role emotional and, mental health). We also examined within group changes for each subscale during the study. Friedman tests found that both groups reported statistically significant improvements in physical functioning scores over the duration of the study, with rehabilitation clients reporting on average an eight and a half point increase (Chi Sq. $(2,41)=17.06, \mathrm{P}=.001)$ in comparison to an average three and a half point increase among usual service users (Chi Sq. $(2,41)=7.12, \mathrm{P}=.028)$. Improvements over time were noted for both groups on the subscales of 'role physical', 'general health', 'vitality', and 'mental health', though these were not statistically significant. Rehabilitation service users reported a reduction in 'bodily pain' scores over the course of the study whilst users of usual services reported an increase in bodily pain scores though statistically significant between group differences were not found. Usual service users reported higher scores for the 'role emotional' scale on admission to the service indicating better outcomes when first assessed though no statistically significant differences were found for this subscale at either of the subsequent time points.

\section{Quality of Life}

Overall, Quality of Life (QoL) improved for both groups over the course of the study (see Table 2). At the time of admission, both groups recorded similar low QoL scores. Mann-Whitney tests found that older people who received the rehabilitation service reported a statistically significant improvement in their QoL by the time of the second interview compared to users of usual services $(z=-2.95, \mathrm{P}=.003)$. Similarly, rehabilitation users reported significantly better QoL scores than users of usual services at the six-month follow-up time point $(z=2.46, \mathrm{P}=.014)$. Friedman tests showed that overall, rehabilitation users reported a significant improvement in QoL scores over the duration of the study Chi Sq. $(2,43)=39.22, \mathrm{P}=.001)$; this improvement occurred during the first eight to ten weeks of the scheme and was maintained at six months. Users of usual services reported an improvement albeit not statistically significant (Chi Sq. $(2,43)$ $=2.02, \mathrm{P}=.365$.

\section{Service Utilization and Cost}

Information was collected from participants on the types of services used during the six-month period since admission and the amount of care provided by each of these services. Generally there were few differences between the services provided to rehabilitation service users and users of usual care. Information on the care management packages of both groups was obtained (see Table 3). Mann-Whitney tests showed that on average, usual service users received significantly more expensive care management packages that rehabilitation service users ( $£ 2344.54$ vs. $£ 6918.81(z=-7.13, P$ $=.001)$ ). It should be noted that 25 rehabilitation clients did not receive any care-managed services in the six months 
Table 1. Change in Functional Measures Over Time

\begin{tabular}{|c|c|c|c|c|}
\hline Assessment & Rehabilitation Service & Usual Service Users & Sig & $95 \% \mathrm{CI}$ \\
\hline \multicolumn{5}{|l|}{ FIM on admission 5} \\
\hline Mean (SD) & $71.15(14.73)$ & $74.71(18.41)$ & $P=.263^{1}$ & 69.78 to 76.05 \\
\hline Median & 73.50 & 73.00 & & \\
\hline Range & $23.00-108.00$ & $33.00-113.50$ & & \\
\hline \multicolumn{5}{|l|}{ FIM at 8-10 weeks ${ }^{5}$} \\
\hline Mean (SD) & $83.13(17.83)$ & $74.47(17.68)$ & $P=.014^{1}$ & 75.54 to 82.55 \\
\hline Median & 84.75 & 72.25 & & \\
\hline Range & $21.00-118.00$ & $33.00-112.00$ & & \\
\hline \multicolumn{5}{|l|}{ FIM at 6 months ${ }^{5}$} \\
\hline Mean (SD) & $83.64(21.08)$ & $78.32(19.21)$ & $P=.206^{1}$ & 76.99 to 85.31 \\
\hline Median & 85.50 & 79.00 & & \\
\hline Range & $34.00-120.00$ & $31.00-113.00$ & & \\
\hline Sig & $\mathbf{P}=.001^{2}$ & $P=.853^{2}$ & & \\
\hline \multicolumn{5}{|l|}{ CDI on admission ${ }^{5}$} \\
\hline Mean (SD) & $49.82(15.10)$ & $49.45(21.01)$ & $P=.865^{3}$ & 46.55 to 54.85 \\
\hline Median & 50.00 & 50.00 & & \\
\hline Range & $15.00-90.00$ & $0.00-90.00$ & & \\
\hline \multicolumn{5}{|l|}{ CDI at 8-10 weeks ${ }^{5}$} \\
\hline Mean (SD) & $63.21(20.35)$ & $49.70(20.59)$ & $\mathbf{P}=.001^{3}$ & 60.93 to 71.07 \\
\hline Median & 65.00 & 50.00 & & \\
\hline Range & $10.00-95.00$ & $0.00-85.00$ & & \\
\hline \multicolumn{5}{|l|}{ CDI at 6 months ${ }^{5}$} \\
\hline Mean (SD) & $62.60(25.12)$ & $56.02(21.93)$ & $P=.144^{3}$ & 55.46 to 69.74 \\
\hline Median & 65.00 & 55.00 & & \\
\hline Range & $10.00-100.00$ & $5.00-95.00$ & & \\
\hline Sig & $P=.001^{4}$ & $P=.006^{4}$ & & \\
\hline \multicolumn{5}{|l|}{ EASY on admission ${ }^{6}$} \\
\hline Mean (SD) & $61.55(12.72)$ & $60.53(17.15)$ & $P=.721^{1}$ & 56.97 to 62.77 \\
\hline Median & 62.00 & 63.00 & & \\
\hline Range & $25.00-84.00$ & $29.00-98.00$ & & \\
\hline \multicolumn{5}{|c|}{ EASY at 8-10 weeks ${ }^{6}$} \\
\hline Mean (SD) & $48.20(17.79)$ & $60.90(17.28)$ & $P=.001^{1}$ & 47.96 to 55.04 \\
\hline Median & 48.00 & 63.00 & & \\
\hline Range & $12.00-95.00$ & $29.00-98.00$ & & \\
\hline \multicolumn{5}{|l|}{ EASY at 6 months ${ }^{6}$} \\
\hline Mean (SD) & $46.22(19.97)$ & $57.32(17.14)$ & $P=.005^{1}$ & 47.44 to 55.39 \\
\hline Median & 43.50 & 55.00 & & \\
\hline Range & $6.00-95.00$ & $28.00-90.00$ & & \\
\hline Sig & $\mathrm{P}=.001^{2}$ & $P=.771^{2}$ & & \\
\hline
\end{tabular}

FIM = Functional Independence Measure; CDI = Community Dependency Index; EASY = EASY-Care Questionnaire (Northern Ireland version)

${ }^{1}$ Independent t-test; ${ }^{2}$ Repeated measures ANOVA; ${ }^{3}$ Mann-Whitney U test; ${ }^{4}$ Friedman test; ${ }^{5}$ High scores indicate improved outcomes; ${ }^{6}$ Low scores indicate improved outcomes 
Table 2. Patient Generated Index QoL Scores

\begin{tabular}{|c|c|c|c|c|}
\hline Assessment $^{3}$ & Rehabilitation Service & Usual Service Users & $\mathrm{Sig}^{1}$ & $95 \% \mathrm{CI}$ \\
\hline \multicolumn{5}{|l|}{ Admission } \\
\hline Mean (SD) & $18.70(18.11)$ & $18.28(20.66)$ & $P=0.588$ & 16.53 to 24.85 \\
\hline Median & 15.00 & 10.00 & & \\
\hline Range & $0.00-80.00$ & $0.00-100.00$ & & \\
\hline Mean (SD) & $38.32(26.20)$ & $22.96(19.03)$ & $P=0.003$ & 27.28 to 37.41 \\
\hline Median & 40.00 & 20.00 & & \\
\hline Range & $0.00-100.00$ & $0.00-70.00$ & & \\
\hline Median & 45.00 & 22.50 & & \\
\hline Range & $0.00-87.50$ & $0.00-80.00$ & & \\
\hline $\mathrm{Sig}^{2}$ & $P=0.001$ & $P=0.365$ & & \\
\hline
\end{tabular}

${ }^{1}$ Mann-Whitney U test $;{ }^{2}$ Friedman test $;{ }^{3}$ Higher scores indicate improved quality of life

Table 3. Type of Service Package Provided and Associated Costs During the Six Months Following Admission

\begin{tabular}{|c|c|c|c|c|}
\hline Service & Rehabilitation Service & Usual Service Users & $\mathrm{Sig}^{1}$ & $95 \% \mathrm{CI}$ \\
\hline \multicolumn{5}{|c|}{ Care-managed Package (£) } \\
\hline Mean (SD) & $2344.54(2884.72)$ & $6918.81(2155.93)$ & $\mathrm{P}=.001$ & -5503.79 to- 3644.75 \\
\hline Median & 1097.60 & 7580.11 & & \\
\hline Range & $.00-9938.50$ & $1386.00-10703.00$ & & \\
\hline Mean (SD) & $1546.70(817.72)$ & & & \\
\hline Median & 1378.67 & & & \\
\hline Range & $354.49-3779.20$ & & & \\
\hline Median & 2848.23 & 6791.82 & & \\
\hline Range & $354.49-13244.15$ & $1386.00-10703.00$ & & \\
\hline \multicolumn{5}{|c|}{$\begin{array}{l}\text { Total 'package' costs for all } \\
\text { service inputs }(\mathfrak{l})^{3}\end{array}$} \\
\hline Mean (SD) & $3864.10(3188.38)$ & $6918.81(2155.93)$ & $\mathrm{P}=.001$ & -4046.96 to- 2062.46 \\
\hline Median & 2883.56 & 7580.11 & & \\
\hline Range & $354.49-13244.15$ & $1386.00-10703.00$ & & \\
\hline
\end{tabular}

${ }^{1}$ Mann-Whitney $U$ test $;{ }^{2}$ Total costs for all participants except those who received residential or nursing home care; ${ }^{3}$ Total costs for all clients including those who received residential or nursing home care 
Table 4. Carers Perception of Strain (CSI)

\begin{tabular}{|c|c|c|c|}
\hline & Rehabilitation Carers & Usual Service Carers & $\mathrm{Sig}^{2}$ \\
\hline Mean (SD) & $4.27(2.62)$ & $5.40(2.23)$ & \\
\hline Median & 4.00 & 5.50 & $P=0.134$ \\
\hline Range & $0-11$ & $3-11$ & \\
\hline Mean (SD) & $3.59(2.28)$ & $5.87(2.25)$ & \\
\hline Median & 3.50 & 6.00 & $\mathbf{P}=\mathbf{0 . 0 0 7}$ \\
\hline Range & $0-7$ & $2-10$ & \\
\hline Median & 3.00 & 6.50 & $P=0.006$ \\
\hline Range & $0-8$ & $3-11.00$ & \\
\hline $\mathrm{Sig}^{3}$ & $P=0.361$ & $P=0.976$ & \\
\hline
\end{tabular}

${ }^{1}$ Higher scores indicate more strain; ${ }^{2}$ Mann-Whitney $U$ test; ${ }^{3}$ Friedman test

following admission to the rehabilitation service. Further analysis showed that 18 rehabilitation users received 'conventional' (non-care managed) services or did not require any services. Seven rehabilitation clients did not receive a care-managed service as they were readmitted to hospital and subsequently died. It should be noted that approximately $30 \%(17 / 56)$ of care managed clients died by the six-month follow-up point.

The costs associated with the rehabilitation service are detailed in Table 3. These costs were calculated from 'contact records' held in the patient's file which record the amount of contact rehabilitation assistants and key therapists had with each individual client. PSSRU unit costs were used to calculate rehabilitation service costs, the average cost of which was $£ 1546.70$. This figure varied depending on the length of time an older person spent on the rehabilitation service and the number of 'inputs' they received. Care costs (excluding residential or nursing home care) were calculated. Users of usual care received a significantly more expensive 'non residential' care package than rehabilitation users ( $£ 3743.24$ vs. $£ 6540.02(z=-5.61, \mathrm{P}=.001))$. The total costs of all care received is also presented in Table 3 . In the six months following admission clients who received usual care, on average received a more expensive package of care than rehabilitation service users ( $£ 3864.10$ vs. $£ 6918.81)$ this difference was again statistically significant $(\mathrm{t}(115)=-6.04$, $\mathrm{P}=.001)$ ).

\section{Carers}

On the whole, carers both of rehabilitation users and users of usual care appeared to be similar in terms of their age, sex, relationship to service users and length of time spent caring. On admission both groups of carers reported similar levels of stress and strain as measured by the Carer Strain
Index (see Table 4). However, when carers were reassessed eight to ten weeks later Mann-Whitney tests indicated that usual care carers reported significantly higher levels of stress when compared to rehabilitation carers $(z=-2.69, \mathrm{P}=.007)$. This pattern of scores was repeated again six months following admission to services $(z=-2.76, \mathrm{P}=.006)$. It is important to note that this statistic was calculated on the basis of reports provided by only 38 carers ( 22 of whom were rehabilitation carers) at the first follow up interview and 31 carers (17 of whom were rehabilitation carers) at the six-month follow up interview. The health status of carers was assessed using the SF-36 questionnaire however; carers did not report any statistically significant differences regarding their health status during the course of the study. Carer QoL was assessed using the carer generated index. Carers from both groups reported similar levels of QoL during the first assessment (see Table 5). During the second assessment rehabilitation carers reported significantly better QoL than usual care carers $(z=-2.40, \mathrm{P}=.017)$. During the six-month assessment rehabilitation carers again reported significantly higher QoL scores than their usual care counterparts $(z=-$ 2.30, $\mathrm{P}=.021)$.

\section{DISCUSSION}

Overall, this study aimed to examine the benefits of a home-based rehabilitation service for frail elderly people in terms of improving functioning, increasing quality of life and reducing admissions and related expenditure to care management. Results show that, by and large, the rehabilitation service offers a safe, feasible alternative to traditional forms of care-managed service. In addition, the service appears to improve decision making about the future care needs of older people by helping them to achieve maximum functioning before decisions about heir long-term care are 
Table 5. Carer Generated Index (CGI)

\begin{tabular}{|c|c|c|c|}
\hline & Rehabilitation Carers & Usual Service Carers & $\mathrm{Sig}^{2}$ \\
\hline Median & 100.00 & 50.00 & $P=0.317$ \\
\hline Range & $0-100$ & $0-100$ & \\
\hline $6-10$ weeks & $(n=22)$ & $(n=16)$ & \\
\hline Mean (SD) & $71.48(35.96)$ & $42.28(29.65)$ & \\
\hline Median & 100.00 & 40.00 & $P=0.017$ \\
\hline Range & $0-100$ & $0-100$ & \\
\hline Median & 100.00 & 40.00 & $P=0.021$ \\
\hline Range & $0-100$ & $20-100$ & \\
\hline $\mathrm{Sig}^{3}$ & $P=0.156$ & $P=0.697$ & \\
\hline
\end{tabular}

${ }^{1}$ Higher scores indicate better outcomes; ${ }^{2}$ Mann-Whitney $U$ test; ${ }^{3}$ Friedman test

made. Collectively this evaluation and other studies (Audit Commission, 1997; Widen-Holmqvist et al., 1998; Miller et al., 2005) provide a growing evidence base to support this model of rehabilitation as a way of increasing client choice, reducing pressure on care management and, generally improving professional decision making and the quality of services provided to older people.

\section{Comparing Rehabilitation Service Users with Clients of Usual Services}

Rehabilitation clients and clients of usual care were similar at admission on a range of key variables. In particular, both groups recorded similar scores on three different standardized and valid professional assessments of functioning. A statistically significant improvement in functioning was observed during the second assessment for rehabilitation clients compared to older people who received usual care. For example, the average level of functioning of the rehabilitation group increased by approximately 12 points on the FIM compared to no change in the scores of the usual care group. This increase in functioning, displayed by rehabilitation clients, was maintained at the time of the six-month assessment. At this time, usual care clients had recorded, on average, a four-point increase in their FIM score. Although this illustrates an improvement in the functional ability of usual care clients it remains somewhat short of the average functional gain experienced by rehabilitation clients. In addition, rehabilitation users recorded statistically significant improvements over time on all three functional measures whilst a significant improvement in the usual care group was observed using the CDI measure only. This finding was fur- ther supported by multivariate analysis of covariance which controlled statistically for any variance between the two groups at the time of admission and found that rehabilitation service users performed significantly better than the usual care group during subsequent assessments.

The results of the comparative analysis of functioning (using three valid measures) provides strong support for the view that participation in the rehabilitation service helps to improve functional ability and achieves this more quickly than existing care management services. It should be noted that the functional ability of usual care clients also appeared to improve between the second and third assessment (though this was not statistically significant); the functioning of clients who had received the rehabilitation service appears to plateau during this period. Therefore, it is reassuring that, on average, all clients appear to improve over time irrespective of the service they received, though the extent to which these trends in levels of functioning would be observed over a longer period of time remains unclear. However, it should be noted that previous studies of home-based rehabilitation found that functional and psychological gains made during the rehabilitation period were maintained up to 12 months later (Cunliffe et al., 2004). Importantly, the comparatively rapid improvement in functioning observed among rehabilitation clients tends to lengthen the time available for professional decision making about future care needs and to achieve this at a key point in the care pathway of clients.

The methodology placed considerable emphasis on gathering data about the views and perspectives of patients. For example, the service users self-reported health status was recorded using the SF-36 questionnaire. Few differences 
were noted between rehabilitation service users and users of usual services at any of the assessment time points. The usual care group reported significantly better scores on the scales relating to 'social functioning' and 'emotional' role at admission indicating that their physical or emotional condition had less impact on their social and daily activities compared to rehabilitation service users. It is unclear why the two groups differed on these scales during the baseline assessment. However, there were no statistically significant differences between the two groups when they were reassessed during subsequent interviews. Both groups reported improvements in perceived physical functioning over the duration of the study; this agreed with the professional assessments of functioning and mirrored similar findings from a previous study that employed the SF-36 to assess older people who were receiving home-based rehabilitation (Mayo et al., 2000). Users of usual care also reported significant improvements over the six-month period in the SF-36 scales relating to 'bodily pain' and 'emotional' role indicating that there was a self-perceived reduction in pain and that emotional problems impacted less on their ability to perform ADLs. However, results relating to the SF-36 questionnaire should be interpreted with caution given the ongoing debate, which questions the extent to which the SF-36 is a valid measure of self-perceived health status in older people (Hill et al., 1996; Walters et al., 2001; Seymour et al., 2001).

Improving the QoL of service users was another objective of the new service and the Patient Generated Index was employed to assess QoL from the Clients' perspective. The important areas of life and functioning, which impacted on the QoL of both, groups included (deterioration in) mobility, independence, and (ability to undertake) activities of daily living (such as toileting, washing or dressing and completing household tasks). Overall, the QoL of rehabilitation service users improved significantly during the study period. Each group recorded a similar low standard of self-perceived QoL at the time of admission. However, the QoL of rehabilitation service users increased significantly compared to the usual care group during subsequent assessments. These results suggest that the rehabilitation service is meeting another key objective in terms of improving the self-perceived QoL of service users and doing so at an early point in their care pathway. Overall, there appears to be a strong relationship between the functional assessments completed by a professional therapist and self-assessed QoL; as the improvements rehabilitation clients made in their functional ability were also reflected in their QoL scores. This finding may be interpreted as providing support for the person centred philosophy of care and active rehabilitation promoted in the Trust's service plan and evidenced in professional practices such as involving clients in establishing rehabilitation goals.

\section{Service Utilization and Costs}

Service use and costs were calculated using the Client Services Receipt Inventory (CSRI) (Beecham \& Knapp, 1992) and PSSRU unit costs (Netten \& Curtis, 2003). Rehabilitation users and users of usual care received similar amounts of hospital and community based services over the duration of the study. The average cost of services other than care management services (as measured by the CSRI) is higher for rehabilitation service users than the usual care group. However, these costs need to be interpreted in the context of other service components such as care management costs.

The costs of the care management service were based on routinely available records provided by the Trust. Usual care clients received significantly more expensive care management packages than rehabilitation service users. On average, the care management costs for the rehabilitation service users were $£ 4574$ cheaper than the costs incurred by the usual care group. Approximately 45 per cent $(25 / 56)$ did not receive a care management service following discharge from the rehabilitation service. A total of 18 people did not need any services or required a non care managed service such as a home help, whilst seven rehabilitation users were readmitted to hospital and subsequently died compared to 30 per cent (17/56) of the usual care group. The reason for the differing death rates in the two groups is unclear and although it would have been interesting to further examine this through survival analysis, the small sample size prevented this.

Approximately 42 per cent (24/56) of usual care clients were placed in residential or nursing homes. Users of usual services continued to receive significantly more expensive care packages than rehabilitation clients even when the costs of nursing/residential care were removed or deducted. In turn, the costs of the rehabilitation package of each client were calculated and added to their care management costs to provide a 'total' cost of care. Users of usual services continued to receive a significantly more expensive package of care even when the costs of the rehabilitation service were included in the calculation. Thus, the results of the analysis appear to provide support for the third major objective of the service, which is to reduce admission to care management and related resources and supports previous research, which found that rehabilitation services were a cost effective alternative to other service options (Miller et al., 2005).

\section{Carers}

It is well recognized now in policy and practice that carers are central to effective community care and thus another main objective of the new rehabilitation service was to ensure that changes in service arrangements did not impact negatively on the health and QoL of carers. The carers of clients in both groups were similar in terms of their age and the length of time they had spent as carers. On admission, 46 carers agreed to take part in the study though fifteen carers were unavailable at either the second time point (9) or the six-month assessment (6). Eleven carer interviews did not take place as the elderly person receiving the service had been readmitted to hospital or had died, while five carers were unavailable or unwilling to complete a subsequent interview. Both groups of carers reported, on average, similar low levels of strain at admission. However, considerable variation was noted in the scores recorded by both groups with some carers reporting very high levels of strain at admission. Rehabilitation service carers reported significantly lower levels of strain than usual care carers during the second interview and this pattern of results was repeated sixmonths following the initial assessment. Overall, the strain scores provided by rehabilitation carers decreased during the 
course of the study though this reduced level of strain was not statistically significant.

There were few differences in terms of health status between the two groups of carers as measured by the SF-36. The majority of carers felt that their caring role had a negative impact on their QoL. For example, caring restricted their freedom or prevented them from taking part in social activities or participating in hobbies. However, some carers believed that caring had little effect on their QoL. When first assessed both groups reported similar levels of QoL. Carers whose relative received the rehabilitation service reported significantly better levels of QoL when subsequently interviewed. In addition, rehabilitation service carers reported an improvement in QoL during the course of the study although this improvement was not statistically significant. Overall and similar to previous research (Miller et al., 2005) the rehabilitation service appears to provide appropriate levels of support for carers as well as clients; and this way of organizing and providing services appears to alleviate carer stress and strain and improve QoL.

\section{CONCLUSION}

The evidence from this evaluation appears to demonstrate that the rehabilitation service is meeting its objectives in terms of improving the functioning of older clients, helping to improve their QoL and reducing care management expenditure. Furthermore, the carers of clients who receive the rehabilitation service also appear to benefit in terms of improved QoL and a reduction in their experience of stress and strain. Therefore, this new service seems to represent for many older people, a safe and feasible alternative to conventional care managed services. However, it is important to recognize the limitations of this study. Due to ethical concerns it was not possible to carry out a randomized controlled trail of the service and a non-randomized design was employed. As a consequence, there may be underlying differences between the two groups which could influence subsequent results. For example, participation in the rehabilitation scheme was voluntary and clients may have been more motivated that other older people receiving a care managed service. In addition, rehabilitation clients were aware that they were participating in a new service which aimed to improve their functioning and help to maintain them in their own homes, again this may have added to their motivation to succeed. A limited number of places were available in the rehabilitation service and it is possible that clients were selected from the population of care managed clients on the basis that they were motivated to succeed or would be more likely to comply with the demands of the new service. Furthermore, a wide range of factors may influence someone's health and it is possible that the two groups varied systematically on other influential factors which were not considered by the research. However, taking these methodological weaknesses into account it appears that this model of community rehabilitation based on a therapist led team of skillsharing therapists supported by well-trained rehabilitation aides appears to be more effective in terms of improvements to functional status and quality of life and appears to be more cost effective than conventional care managed services. Demand for this service is evidenced by the fact that since its inception the rehabilitation service has expanded on several occasions to meet an increasing demand for communitybased rehabilitation. The results from this study suggest that community-based rehabilitation is an appropriate service response for many older people and has an important role to play in the continuum of services necessary to meet their diverse care needs.

\section{ACKNOWLEDGEMENT}

We would like to thank the staff of the South and East Belfast Trust Elderly Programme of Care for their help and support throughout this study. The views expressed are those of the authors".

\section{REFERENCES}

Audit Commission. (1997). The Coming of Age: Improving Care Services For Older People. London: Audit Commission.

Beecham, J., \& Knapp, M. (1992). Costing Psychiatric Interventions. In Thornicroft GJ, Brown CR, Wing J (Eds). Measuring Mental Health Needs. Oxford: University Press.

Clisset, P. (2001). Making the Transition to Long-Term Care. Professional Nurse, 16(9), 1363-6.

Cunliffe, A., Gladman, J., Husbands, S., et al. (2004). Sooner and Healthier: A Randomised Controlled Trial and Interview Study of an Early Discharge Rehabilitation Service for Older People. Age and Ageing, 33, 246-252.

Dempster, M., \& Donnelly, M. (2000). How well do Elderly People Complete Individualised Quality of Life Measures: An Exploratory Study. Quality of Life Research, 9(4), 369-375.

Eakin P, \& Baird, H. (1995). The Community Dependency Index: A Standardized Measure of Outcome for Community Occupational Therapy. British Journal of Occupational Therapy, 58(1), 17-22.

Giustis, A., Barone, A., Oliveri, M., Razzano, M., Palummeri, E., \& Pioli, G. (2006). An Analysis of the Feasibility of Home Rehabilitation Among Elderly people with Proximal Femoral Fractures. Archives of Physical and Medical rehabilitation, 87(6), 826-831.

Hill, S., Harries, U., \& Popay, J. (1996). Is the Short Form 36 (SF-36) Suitable for Routine Health Outcomes Assessment in Health Care for Older People? Evidence from Preliminary Work in Community Based Services in England. Journal of Epidemiology and Community Health, 50(1), 94-98.

Keith, R., Granger, C., Hamilton, B., \& Sherwin, F. (1987). The Functional Independence Measure: A new tool for Rehabilitation. Advanced Clinical Rehabilitation, 1, 6-18.

Mayo, N., Wood-Dauphinee, S., \& Cote, R. et al. (2000). There's No Place like Home: An Evaluation of Early Supported Discharge for Stroke. Stroke, 31(5), 1016-1023.

Melis, R., Rikkart, M., Parker, S., \& Van Eijken, M. (2004). What is Intermediate Care? British Medical Journal, 329, 360-361

Miller, P., \& Cunliffe, A., Husbands, S., et al. (2005). Economic Analysis of an Early Discharge Rehabilitation Service for Older People. Age and Ageing, 34(3), 274-280.

Netten, A., \& Curtis, L. (2003). Unit Costs of Health and Social Care. University of Kent: PSSRU.

NHS. (2000). The NHS Plan: The Government's Response to the Royal Commission on Long Term Care. London: The Stationary Office.

NHS. (2003). The National Service Framework for Older People. London: The Stationary Office.

Philp, I., Armstrong, G., Coyle, G., et al. (1998). A Better Way to Measure Disability in Older People. Age and Ageing, 27, 519-522.

Reed, I., \& Morgan, J. (1990). Discharging Older People from Custody to Care Homes: Implications for Nursing. Journal of Advanced Nursing, 29(4), 819-825.

Robinson, B.C. (1983). Validation of a Caregiver Strain Index. Journal of Gerontology, 38, 344-353.

Seymour, G., Russell, E., Garratt, A. (2001). Problems in using Health Survey Questionnaires in Older Patients with Physical Disabilities. The Reliability and Validity of the SF-36 and the Effect of Cognitive Impairment. Journal of Evaluation in Clinical Practice, 7(4), 411-418.

Thomas, S. \& MacMahon, D. (2001). Intermediate Care. Journal of Community Nursing, 15(6), 12-20. 
Vincent, C., Deaudelin, I., Robichaud, L., Rosseau, J., Viscogliosi, C., Talbot, L.R., \& Desrosiers, J. (2007). Rehabilitation Needs for Older Adults with Stroke Living at Home: Perceptions of Four Populations. BMC Geriatrics, 13, 7-20.

Walters, S., Munro, J., Brazier, J. (2001). Using the SF-36 with Older Adults: A Cross-Sectional Community-Based Survey. Age and Ageing, 30, 337-343.
Ware, J., \& Sherbourne, D. (1992). The MOS 36-Item Short Form Health Survey (SF-36). Conceptual Framework and Item Selection. Medical Care, 30, 324-328.

Widen-Holmqvist, L., Von Koch, L., Kostulas, V., et al. (1998). A Randomised Controlled Trial of Rehabilitation at Home after Stroke in South West Stockholm. Stroke, 31, 2989-2994.

Wright, K. (2003). Assessment for long-term care: a snapshot of nursing practice. British Journal of Community Nursing, 8(1), 6-14.

(C) Scott and Donnelly; Licensee Bentham Open.

This is an open access article distributed under the terms of the Creative Commons Attribution License (http://creativecommons.org/license/by/2.5/), which permits unrestrictive use, distribution, and reproduction in any medium, provided the original work is properly cited. 